\title{
A satisfação com o suporte social e o funcionamento familiar numa amostra de adolescentes com e sem irmãos
}

\section{Satisfaction with social support and family functioning in a sample of adolescents with and without siblings}

\author{
M. Mendes, I. Relva, O. Fernandes
}

ARTIGO ORIGINAL | ORIGINAL TITLE

\begin{abstract}
RESUMO
A satisfação com o suporte social torna-se essencial para o desenvolvimento dos adolescentes, uma vez que contribui para o bem-estar destes. Esta investigação pretende explorar a satisfação com o suporte social e o funcionamento familiar em adolescentes, com e sem irmãos. A amostra foi constituída por 365 participantes, 213 do sexo feminino e 152 do sexo masculino, com idades compreendidas entre os 15 e os 20 anos. Os instrumentos utilizados foram a Escala de Satisfação com o Suporte Social (ESSS) e a Systemic Clinical Outcome and Routine Evaluation (SCORE-15). Foram realizadas correlações de Pearson e verificou-se uma associação negativa entre o suporte social e o funcionamento familiar, isto é, à medida que o suporte social aumenta, as dificuldades familiares diminuem. Efetuaramse análises de variância multivariada (MANOVA), com vista a analisar as diferenças em relação às variáveis sociodemográficas. Os resultados indicaram que, em função da configuração familiar, verificou-se que adolescentes de famílias com pais casados ou em união de facto apresentam maiores níveis de satisfação com a família comparativamente aos adolescentes com os pais divorciados. No funcionamento familiar verificou-se que os adolescentes com pais divorciados apresentam maiores dificuldades de recursos familiares, de comunicação na família e de dificuldades familiares. Em relação ao sexo, não se verificaram diferenças significativas. Finalmente, não se encontraram diferenças entre adolescentes filhos únicos e os que têm irmãos, relativamente à satisfação com o suporte social, bem como no funcionamento familiar. Assim, sublinha-se a importância de um bom funcionamento familiar, no sentido de contribuir para a qualidade das relações dos adolescentes.

Palavras-chave: suporte social, funcionamento familiar, adolescência, irmãos, família
\end{abstract}

\section{ABSTRACT}

Satisfaction with social support becomes essential for the development of adolescents, as it contributes to their well-being. This research explores satisfaction with social support and family functioning in adolescents with and without siblings. The sample consisted of 365 participants, 213 were female, and 152 were male, aged between 15 and 20. The instruments used were the Social Support Satisfaction Scale (ESSS) and the Systemic Clinical Outcome and Routine Evaluation (SCORE-15). Pearson correlations were performed, and there was a negative association between social support and family functioning. That is, support increases, family difficulties decrease. Differential analyzes were performed using multivariate analysis of variance (MANOVA) to verify differences in sociodemographic variables. The results indicated that depending on the family configuration, adolescents from families with married or de facto parents have higher levels of family satisfaction than adolescents with divorced parents. In family functioning, adolescents with divorced parents have greater difficulties in family resources, family communication, and family difficulties. Regarding gender, no significant differences were found. Finally, we investigated and found no differences between adolescent-only children and siblings regarding satisfaction with social support and family functioning. Thus, the importance of good family functioning is emphasized to contribute to adolescents' relationships.

Keywords: social support, family functioning, adolescence, siblings, family 
No período de transição para a vida adulta, o suporte social torna-se fundamental. Este protege e ajuda os indivíduos garantindo-lhes uma melhor transição (Paulsen \& Berg, 2016) em momentos de dificuldade, uma vez que estes recorrem a figuras significativas, tais como, familiares, amigos, colegas, vizinhos e professores (Matsumoto et al., 2017; Thompson, 2015) para ajudá-los a resolver problemas (Chen et al., 2017) e para fornecer ajuda psicológica, física e financeira (Mattson \& Hall, 2011). Enquanto alguns jovens irão lidar com a transição da adolescência para a vida adulta sem qualquer dificuldade, muitos deles precisarão de assistência, apoio e suporte social para garantir uma melhor evolução (Paulsen \& Berg, 2016).

O período da adolescência é caracterizado pela expansão dos grupos de pares e da modificação da influência de cada um, uma vez que ocorrem mudanças nas relações com os outros, nomeadamente com os pais e pares. Devido à estreita interdependência que se estabelece entre os adolescentes e o grupo de pares, esta influência é crucial no desenvolvimento destes, na qual parece destacar-se a importância do apoio emocional, lealdade, compreensão e intimidade (Antunes \& Fontaine, 2005). Os adolescentes procuram a partilha de experiências e o apoio do grupo de pares, o que representa um suporte relevante para o bem-estar físico e psicológico destes (Guerreiro \& Sampaio, 2013).

\section{Suporte social}

O suporte social diz respeito a qualquer tipo de comunicação, verbal ou não-verbal, que ajuda os indivíduos a sentirem-se seguros em relação a uma situação. As principais características desta definição de suporte social são o trabalho em rede, a ajuda psicológica, física e financeira (Mattson \& Hall, 2011).

Assim, o suporte social pode ser definido como recursos emocionais, instrumentais e informacionais que os indivíduos obtêm através dos seus amigos e/ou familiares
(Sangalang \& Gee, 2012; Matsumoto et al., 2017) que lhes fornecem uma certa ajuda para adotar ou manter um comportamento. Sendo os amigos e/ou familiares que contribuem para tal suporte social, um estudo indicou que estudantes universitários relatam passar mais tempo com os seus amigos do que com os seus familiares, e que é possível que os amigos tenham mais oportunidades de discutir, apoiar e adotar certos comportamentos a serem seguidos (Belanger \& Patrick, 2018).

Coob (1976) define suporte social como a percepção que o sujeito tem de que é amado, de que pertence a uma rede de comunicação e de obrigações mútuas, e que o leva a crer que é apreciado e valorizado. O suporte social é, assim, considerado benéfico para a saúde dos indivíduos e promove a saúde mental, assim como a saúde física dos mesmos (Sangalang \& Gee, 2012) contribuindo para a promoção do bem-estar (Mühlen et al., 2013). O suporte social não auxilia apenas no facto de o indivíduo se sentir melhor a lidar com os desafios, como também leva a uma melhoria do bem-estar em geral, significando que ter acesso a qualquer tipo de suporte social é essencial para uma vida saudável. Este associa-se a vários resultados de saúde que incluem a recuperação de doenças e a redução da mortalidade. Uma explicação relacionada com os benefícios do suporte social refere que se os indivíduos tiverem uma rede de apoio, torna-se suficiente para se manterem saudáveis ou recuperarem de alguma doença (Mattson \& Hall, 2011).

Considera-se também importante distinguir o suporte social percebido e o oferecido/recebido. Essa diferença é ainda mais importante durante a adolescência, período em que podem surgir discrepâncias entre o que o adolescente está a receber e o que o adolescente percebe que está a receber (Adler-Constantinescu, Besu, \& Negovan, 2013). O suporte social oferecido/ recebido é o suporte que um indivíduo recebe em termos do que é dito, do que é dado e do que é feito para esse indivíduo. No entanto, 
muito mais significativo do que o suporte social oferecido/recebido é a percepção individual da disponibilidade de suporte. O suporte percebido refere-se à crença individual de que o suporte social está disponível, é geralmente considerado positivo ou negativo e fornece o que é considerado necessário para aquele indivíduo (Mattson \& Hall, 2011).

Recentemente, um estudo indicou que existem diferenças de sexo na percepção do suporte social, sendo que os indivíduos do sexo feminino perceberam maior suporte social do que os do sexo masculino (Alves \& Dell'Aglio, 2015).

\section{Tipos de suporte social}

Existem três tipos de suporte social, o suporte emocional, o suporte instrumental e o suporte informacional. O primeiro, o suporte emocional, é a comunicação que responde às necessidades emocionais ou afetivas de um indivíduo, ou seja, são expressões que transmitem cuidado e preocupação. Expressões de suporte emocional não tentam resolver diretamente um problema, mas servem para elevar o humor de um indivíduo. O próximo tipo de suporte social, o suporte instrumental, reforça o indivíduo na sua capacidade de lidar com um problema ou realizar uma tarefa necessária. Este tipo de suporte incentiva os indivíduos a tomarem as ações necessárias e a convencê-los de que têm capacidade para enfrentar determinados problemas. Por último, o suporte informacional diz respeito à comunicação que fornece informações úteis ou necessárias, uma vez que ao enfrentar qualquer situação, muitas vezes são necessárias informações para tomar decisões (Mattson \& Hall, 2011).

Considerando os vários tipos de suporte social, poderá surgir a pergunta "Qual o melhor tipo de suporte social?”. Essa resposta poderá ser respondida através da Theory of Optimal Matching (Cutrona \& Russell, 1990), que propõe que o melhor tipo de suporte social é aquele que corresponde às necessidades de um indivíduo.

\section{Relações fraternais}

Os relacionamentos entre irmãos têm características por um lado semelhantes e, por outro, diferentes dos relacionamentos entre pais-filhos e dos relacionamentos entre os pares. Assim, e à semelhança do relacionamento pais-filhos, o relacionamento entre os irmãos caracteriza-se por fortes laços emocionais, sobretudo quando os irmãos tendem a ter uma idade mais próxima entre si, semelhante aos relacionamentos entre pares (Cox, 2010). As relações fraternais contribuem para o desenvolvimento individual. Estas relações podem ser tanto próximas como distantes, mais ou menos harmoniosas ou conflituosas. No entanto, quanto menor for a diferença de idades, mais a interação entre eles se estrutura numa reciprocidade ativa (Fernandes, 2002).

Os irmãos tornam-se cuidadores, amigos e figuras de suporte ao longo da vida, assim como proporcionam apoio emocional e a partilha de experiências. Assim, os irmãos mais velhos tornam-se modelos para os mais novos, uma vez que estes costumam imitar os seus comportamentos (Fernandes, 2005). São importantes na fase da adolescência, pois influenciam a formação de identidade dos irmãos mais novos (Wong et al., 2010). Segundo Fernandes (2005), os filhos únicos possuem uma menor capacidade de se relacionarem com os outros pela ausência de irmãos e falta de experiência estabelecida com estes.

\section{Funcionamento familiar}

O funcionamento familiar diz respeito aos processos estruturais e organizacionais das famílias (Berge, Wall, Larson, Loth, \& Neumark-Sztainer, 2013), abarcando as relações intrafamiliares e a própria dinâmica familiar, isto é, como cada membro da família interage com os outros membros, ou seja, compreende as complexas interacções interpessoais do grupo familiar (Prioste, Tavares, \& Magalhães, 2019), engloba a comunicação 
familiar (Lamb et al., 2016; Tolou-Shams et al., 2018) e características tais como a coesão familiar e a qualidade das relações. A coesão pode ser definida como o nível de proximidade e de união entre os elementos da família (Schulte et al., 2017). Famílias coesas têm apoio e compreensão mútua que se reflete entre os membros da família. Logo, o efeito das relações familiares é muito importante, uma vez que uma boa relação dentro da própria família, isto é, uma comunicação adequada entre os membros, tais como pais e irmãos, leva a menos conflitos. Nestas famílias, os seus membros encontram formas de resolução dos problemas e torna-se fundamental para que cada membro se sinta valorizado (Emam \& Abu-Serei, 2014).

Assim, de forma geral, o funcionamento familiar pode ser definido como o processo através do qual a família garante a satisfação das suas necessidades, toma decisões, estabelece regras e define objetivos que favoreçam conjuntamente o desenvolvimento familiar e o dos indivíduos que a compõem. São considerados aspetos essenciais do funcionamento no dia-a-dia das famílias: os recursos familiares, a comunicação da família e as dificuldades a que a família se encontra sujeita. É através da junção destes três aspetos que a funcionalidade da família é definida (Stratton, Bland, Janes, \& Lask, 2010).

Num estudo recente, em que se procurou explorar diferenças em função do sexo dos adolescentes quanto ao funcionamento familiar, constatou-se que os adolescentes de ambos os sexos percebiam o funcionamento familiar de forma similar (Coppari, Eguiarte, Zavala, \& Bagnoli, 2017).

Funcionamento familiar e suporte social

A família pode influenciar e ser influenciada pelo suporte social, uma vez que as relações que se criam podem ser consideradas como fatores protetores para os membros da família. Durante a adolescência, o papel da família torna-se fundamental devido às mudanças que ocorrem no suporte social. Deste modo, verificou-se que o suporte social influencia o funcionamento familiar (Seibel et al., 2017).

Torna-se importante estudar a relação entre estas duas variáveis, o funcionamento familiar e o suporte social, uma vez que ambos são considerados aspectos determinantes para o bem-estar e para a construção da resiliência, pois auxiliam na resolução de problemas e conflitos e são factores protectores para o desenvolvimento individual (Seibel et al., 2017).

Com este estudo pretende-se conhecer melhor o papel de variáveis como o suporte social e o funcionamento familiar na adolescência, e analisar o papel pouco explorado da fratria nesta fase da vida que é a transição para a vida adulta. Sabe-se que a estrutura e o funcionamento familiar são muito significativos nesta fase da vida dos adolescentes, uma vez que criam condições para o desenvolvimento, maturação e autonomia destes (Matejevic et al., 2014). Do mesmo modo, tal como foi descrito anteriormente, as redes de suporte e apoio, familiares e sociais, são importantes, uma vez que os adolescentes procuram segurança, nesta fase da vida (Studzinski, 2016). O presente estudo tem como objectivo geral correlacionar a relação entre o suporte social e o funcionamento familiar em adolescentes com e sem irmãos. Os objectivos específicos são: (a) correlacionar a satisfação com o suporte social e o funcionamento familiar; (b) Comparar por sexo, idade, configuração familiar e número de irmão ao nível do suporte e funcionamento familiar.

\section{MÉTODOS}

O presente estudo tem um carácter transversal, quantitativo e correlacional. Por estudo transversal entende-se que os dados obtidos foram recolhidos num determinado momento; o caráter quantitativo da investigação diz respeito às características mensuráveis das variáveis e que se podem exprimir em valores numéricos; e o carácter correlacional remete para o facto de se estabelecer relações entre as 
variáveis (Almeida \& Freire, 2017).

As variáveis independentes são o sexo, a idade dos adolescentes, a configuração familiar e o número de irmãos. As variáveis dependentes são a satisfação com o suporte familiar e o funcionamento familiar.

\section{Amostra}

A presente investigação foi constituída por 365 adolescentes de ambos os sexos, em que 213 (58.4\%) são do sexo feminino e 152 (41.6\%) do sexo masculino, pertencentes a 2 escolas na região norte de Portugal, com idades compreendidas entre os 15 e os 20 anos.

No que concerne à escolaridade dos adolescentes participantes, os mesmos frequentam entre o $10^{\circ}$ e o $12^{\circ}$ ano, sendo que 110 frequentam o $10^{\circ}$ ano de escolaridade (30.1\%), 152 estudam no $11^{\circ}$ ano $(41.7 \%)$, e 103 estudam no $12^{\circ}$ ano (28.2\%). Encontram-se a frequentar o curso profissional 24 alunos (6.6\%), o curso de ciências e tecnologias 182 alunos (49.9\%), o curso de línguas e humanidades 130 alunos (35.6\%) e o curso de artes visuais 29 alunos (7.9\%).

Os adolescentes vivem, em média, com 2 pessoas $(\mathrm{M}=2.72$, $\mathrm{DP}=.85)$.

Relativamente ao número de irmãos, a presente amostra de adolescentes apresenta em média 1 irmão $(\mathrm{M}=1.23$, $\mathrm{DP}=.420)$, e 83 (22.7\%) adolescentes indicam não ter irmãos. Assim sendo, 210 (57.5\%) têm um irmão, 54 (14.8\%) têm dois irmãos, 14 (3.8\%) mencionam ter três irmãos, e 4 (1.2\%) referem ter quatro ou mais irmãos. Verifica-se que a maioria dos adolescentes 323 (88.5\%) provêm de famílias com os pais casados ou em união de facto, 39 (10.7\%) provêm de famílias com os pais divorciados e $3(.8 \%)$ provêm de famílias com pais solteiros. As habilitações académicas dos pais dos adolescentes variam entre o $4^{\circ}$ ano e o ensino superior, com 34 adolescentes (9.3\%) a indicar que os seus pais têm o $1^{\circ}$ ciclo de ensino básico, e 27 adolescentes (7.4\%) referem que as mães têm esse grau de escolaridade. Com o $2^{\circ}$ ciclo de ensino básico surgem 69 pais de adoles- centes $(18.9 \%)$ e 56 mães $(15.3 \%)$. Com o $3^{\circ}$ ciclo de ensino básico surgem 82 pais $(22.5 \%)$ e 66 mães (18.1\%), e com o ensino secundário há 95 pais (26.0\%) e 93 mães (25.5\%). Com o grau de ensino superior são indicados 85 pais dos adolescentes (23.3\%) e 123 mães (33.7\%).

Quadro 1

Variáveis sociodemográficas.

\begin{tabular}{|c|c|c|}
\hline & $n=365$ & $\%$ \\
\hline \multicolumn{3}{|l|}{ Sexo } \\
\hline Masculino & 152 & $41.6 \%$ \\
\hline Feminino & 213 & $58.4 \%$ \\
\hline \multicolumn{3}{|l|}{ Ano de escolaridade } \\
\hline $10^{\circ}$ ano & 110 & $30.1 \%$ \\
\hline $11^{\circ}$ ano & 152 & $41.7 \%$ \\
\hline $12^{\circ}$ ano & 103 & $28.2 \%$ \\
\hline \multicolumn{3}{|l|}{ Cursos } \\
\hline Curso profissional & 24 & $6.6 \%$ \\
\hline Ciências e Tecnologias & 182 & $49.9 \%$ \\
\hline Línguas e Humanidades & 130 & $35.6 \%$ \\
\hline Artes Visuais & 29 & $7.9 \%$ \\
\hline \multicolumn{3}{|l|}{ Número de irmãos } \\
\hline 0 & 83 & $22.7 \%$ \\
\hline 1 & 210 & $57.5 \%$ \\
\hline 2 & 54 & $14.8 \%$ \\
\hline 3 & 14 & $3.8 \%$ \\
\hline 4 ou mais & 4 & $1.2 \%$ \\
\hline \multicolumn{3}{|l|}{ Configuração familiar } \\
\hline $\begin{array}{l}\text { Pais casados ou em união } \\
\text { de facto }\end{array}$ & 323 & $88.5 \%$ \\
\hline Pais divorciados & 39 & $10.7 \%$ \\
\hline Pais solteiros & 3 & $.8 \%$ \\
\hline \multicolumn{3}{|l|}{ Habilitações pais } \\
\hline $1^{\circ}$ Ciclo ensino básico & 34 & $9.3 \%$ \\
\hline $2^{\circ}$ Ciclo ensino básico & 69 & $18.9 \%$ \\
\hline $3^{\circ}$ Ciclo ensino básico & 82 & $22.5 \%$ \\
\hline Ensino secundário & 95 & $26.0 \%$ \\
\hline Ensino superior & 85 & $23.3 \%$ \\
\hline \multicolumn{3}{|l|}{ Habilitações mães } \\
\hline $1^{\circ}$ Ciclo ensino básico & 27 & $7.4 \%$ \\
\hline $2^{\circ}$ Ciclo ensino básico & 56 & $15.3 \%$ \\
\hline $3^{\circ}$ Ciclo ensino básico & 66 & $18.1 \%$ \\
\hline Ensino secundário & 93 & $25.5 \%$ \\
\hline Ensino superior & 123 & $33.7 \%$ \\
\hline
\end{tabular}


Instrumentos

Questionário sociodemográfico - Este instrumento permitiu recolher informação relativa ao participante quanto a variáveis como a idade, sexo, escolaridade, número de irmãos, número e elementos do agregado familiar, estado civil e escolaridade dos pais, assim como a idade dos mesmos.

A Escala de Satisfação com o Suporte Social (ESSS) de Pais-Ribeiro (1999) é constituída por 15 itens apresentados segundo um conjunto de afirmações para um autopreenchimento. $\mathrm{O}$ indivíduo deve indicar o grau em que concorda com a afirmação numa escala de Likert com cinco possibilidades de resposta: 1- concordo totalmente, 2- concordo na maior parte, 3- não concordo nem discordo, 4- discordo na maior parte e 5- discordo totalmente. Esta escala é dividida em quatro dimensões: satisfação com os amigos e mede a satisfação com estes, inclui os itens de 1 a 5 (e.g., "os amigos não me procuram tantas vezes como eu gostaria"); intimidade e mede a percepção da existência de suporte social íntimo, engloba os itens 6 a 9 (e.g., "Por vezes sinto-me só no mundo e sem apoio"); satisfação com a família e mede a satisfação com o suporte social familiar existente, inclui os itens de 10 a 12 (e.g., "Estou satisfeito com a forma como me relaciono com a minha família") e a última dimensão atividades sociais e mede a satisfação com as atividades sociais que efetua, abrange os itens 13 a 15 (e.g. "Sinto falta de atividade sociais que me satisfação"). O valor mínimo desta escala é de 15 valores e o máximo corresponde a 75 (Pais-Ribeiro, 1999). A análise da consistência interna no estudo original, através do alfa de Cronbach revelou que este apresenta boa consistência interna, na medida em que o seu valor para a escala total foi de (e.g. $\alpha=.85)$. Em relação a cada uma das dimensões, foram obtidos os seguintes valores (e.g. $\alpha=.83$ ) para a dimensão satisfação com os amigos, (e.g. $\alpha=.74$ ) para a dimensão intimidade, (e.g. $\alpha=.74$ ) para a dimensão satisfação com a família, e ainda (e.g. $\alpha=$.64) para a dimensão actividades sociais (Pais-Ribeiro, 1999). No presente estudo, a análise da consistência interna através do alfa de Cronbach revelou que este apresenta boa consistência interna, na medida em que o seu valor para a escala total foi de (e.g. $\alpha=.87$ ). No que respeita a cada uma das dimensões, foram obtidos os seguintes valores: (e.g. $\alpha=.80$ ) para a satisfação com os amigos, (e.g. $\alpha=.69$ ) para a intimidade, (e.g. $\alpha=.79$ ) para a satisfação com a família, e ainda (e.g. $\alpha=$.67) para as actividades sociais. Embora duas dimensões apresentam um valor $<.70$, estes são considerados no limite da confiabilidade adequada, assim como, valores $\geq .70$ são indicadores de confiabilidade aceitável (Marôco, Campos, Vinagre, \& Pais-Ribeiro, 2014).

A Systemic Clinical Outcome and Routine Evaluation (SCORE-15) avalia o funcionamento familiar, tendo sido desenvolvida por Stratton, Bland, Janes e Lask (2010), em Inglaterra. A versão portuguesa foi validada e traduzida por Portugal, Sotero, Cunha, Vilaça e Relvas (2010) é um questionário de auto-resposta composto por 15 itens que avalia vários aspetos do funcionamento familiar distribuídos por três dimensões da família: Recursos Familiares, Comunicação na Família e Dificuldades Familiares. A primeira dimensão refere-se aos recursos e à capacidade de adaptação da família, inclui os itens 1 , $3,6,10$ e 15, a segunda avalia a comunicação no sistema familiar com os itens 2, 4, 8, 12 e 13 e a terceira remete para as dificuldades no sistema familiar, engloba os itens 5, 7, 9, 11 e 14. Os itens são apresentados numa escala de Likert com 5 níveis de resposta: 1 - muito bem; 2- bem; 3- em parte; 4- mal; 5- muito mal. Quanto mais elevadas forem as pontuações do SCORE-15, maiores dificuldades são vividas na família. O SCORE-15 pode ser aplicado aos vários elementos da família com mais de 12 anos. De acordo com a análise da consistência interna, através do alfa de Cronbach da escala 
portuguesa, a escala total apresenta uma boa consistência interna (e.g. $\alpha=.84$ ), tal como acontece com a dimensão Recursos Familiares com (e.g. $\alpha=.85$ ), a dimensão Comunicação na Família com (e.g. $\alpha=.83$ ) e a dimensão Dificuldades Familiares com (e.g. $\alpha=.82$ ) (Vilaça, Silva, \& Relvas, 2014). No presente estudo, de acordo com a análise da consistência interna, através do alfa de Cronbach, a escala total apresentou valores bastante razoáveis (e.g. $\alpha=.87$ ), tal como acontece com a dimensão Recursos Familiares (e.g. $\alpha=.81$ ), a dimensão Comunicação na Família (e.g. $\alpha$ $=.70)$ e a dimensão Dificuldades Familiares (e.g. $\alpha=.68$ ). Embora esta última dimensão apresente um valor $<.70$, este é considerado no limite da confiabilidade adequada, assim como, valores $\geq .70$ são indicadores de confiabilidade aceitável (Marôco et al., 2014).

\section{Procedimentos}

O protocolo do presente estudo foi submetido à avaliação, tendo sido dado parecer positivo pela Comissão de Ética da Universidade de Trás-os-Montes e Alto Douro (UTAD). De seguida, foi necessário submeter um pedido de autorização à Direção-Geral da Educação (DGE) do Ministério da Educação e Ciência. Após a obtenção desta autorização, foi efetuado um contacto com duas escolas do ensino secundário do Norte de Portugal, com vista a obter as devidas autorizações pelos directores das mesmas. Após a obtenção dos compromissos institucionais, oficializados através de um documento escrito, foi solicitado o consentimento informado dos encarregados de educação para a participação dos adolescentes menores, assim como aos alunos maiores de idade foi entregue um consentimento livre, informado e esclarecido. Posteriormente e, apenas depois das autorizações dos encarregados de educação, procedeu-se à aplicação dos questionários, aos alunos do ensino secundário.

A administração dos instrumentos decorreu em sala de aula, com turmas seleccionadas aleatoriamente, durante aproximadamente 20 minutos. As turmas eram compostas por aproximadamente 25 alunos, dispostos dois a dois na sala de aula. Apresentaram-se os objectivos gerais do estudo aos alunos, forneceram-se as instruções necessárias para o preenchimento dos questionários e informou-se a cada um da participação voluntária, bem como da confidencialidade e do anonimato dos dados obtidos.

Estratégias de análise de dados

Inicialmente, foi construída a base de dados para uma posterior análise estatística, com recurso ao software Statistical Package for the Social Sciences - SPSS - versão (25.0) para o sistema Windows.

Após a introdução dos dados no programa estatístico, foi executada a limpeza da base de dados, excluindo-se questionários preenchidos de forma inadequada e/ou incompletos, isto é, exclusão de missings e outliers. Assim, um questionário com mais do que $10 \%$ das respostas em branco foi retirado da amostra.

Posteriormente, foi efectuada a inversão de alguns itens, de acordo com a referência teórica dos autores originais dos instrumentos utilizados.

Após a criação das dimensões referentes a cada instrumento, foram realizadas análises de outliers. Os outliers dizem respeito aos valores que se encontram fora do intervalo médio, e que consequentemente provocam um enviesamento dos resultados (Pallant, 2005; Marôco, 2014). No presente estudo, foram apenas identificados outliers multivariados com base à distância de Mahalanobis. Esta estatística mede a distância de uma observação x à média de todas as observações, de todas as variáveis (Marôco, 2014). Seguidamente realizou-se a análise da consistência interna (alpha de Cronbach). No que respeita à análise dos dados, foram realizadas Correlações de Pearson, podendo as mesmas 
assumir um valor entre -1 e 1. Este coeficiente permite determinar a associação entre variáveis, assim como a força e a intensidade dessa mesma associação. Estas podem ser positivas ou negativas, e ainda apresentar um grau de associação baixo, moderado ou forte (Pallant, 2005). De acordo com Cohen (1988), a correlação é fraca quando $r=.10$ a .29 ou $r=-.10$ a -.29; média quando $r=.30$ a .49 ou $r=-.30$ $\mathrm{a}-.4 .9$ e forte quando $r=.50$ a 1.0 ou $r=-.50$ a -1.0. Recorreu-se às análises de variância multivariada (MANOVAS), com nível de significância de 5\% ( $p \leq .05)$ e calculou-se o eta-quadrado, uma vez que este representa a proporção de variação da variável dependente que é explicada pela variável independente. De acordo com Cohen (1988), os valores para o eta quadrado podem variar de 0 a 1 , sendo que .01 é pequeno; .06 é moderado e .14 é grande.

\section{RESULTADOS}

Analisando a correlação entre o suporte social e o funcionamento familiar (Quadro1), verificou-se que a dimensão satisfação com os amigos correlaciona-se de forma média e negativa com os recursos familiares $(r=-.414 ; p<$ $.01)$, a comunicação na família $(r=-.328 ; p<$ $.01)$ e as dificuldades familiares $(r=-.430 ; p$ $<$.01). A dimensão intimidade apresenta uma correlação média e negativa com as dimensões recursos familiares $(r=-.417 ; p<.01)$, comunicação na família $(r=-.398 ; p<.01)$ e dificuldades familiares $(r=-.442 ; p<.01)$. No que concerne à dimensão satisfação com a família, verifica-se uma correlação forte e negativa com as dimensões recursos familiares $(r=-.599 ; p<$ $.01)$, comunicação na família $(r=-.502 ; p<.01)$ e dificuldades familiares $(r=-.554 ; p<.01)$. Por fim, a dimensão atividades sociais correlaciona-se de forma fraca e negativa com todas as dimensões pertencentes ao questionário respeitante ao funcionamento familiar, nomeadamente recursos familiares $(r=-.267 ; p<.01)$, comunicação na família $(r=-.255 ; p<.01)$ e dificuldades familiares $(r=-.262 ; p<.01)$.

Quadro 2

Correlação entre as dimensões do suporte social e do funcionamento familiar, média e desvio-padrão.

\begin{tabular}{|c|c|c|c|c|c|c|c|}
\hline & 1 & 2 & 3 & 4 & 5 & 6 & 7 \\
\hline 1. Recursos Familiares & - & & & & & & \\
\hline 2. Comunicação na Família & $.600^{* *}$ & - & & & & & \\
\hline 3. Dificuldades Familiares & $.583^{* *}$ & $.656^{* *}$ & - & & & & \\
\hline 4. Satisfação com os Amigos & $-.414^{* *}$ & $-.328 * *$ & $-.430 * *$ & - & & & \\
\hline 5. Intimidade & $-.417^{* *}$ & $-.398^{* *}$ & $-.442^{* *}$ & $.704^{* *}$ & - & & \\
\hline 6. Satisfação com a Família & $-.599 * *$ & $-.502^{* *}$ & $-.554^{* *}$ & $.519 * *$ & $.487^{* *}$ & - & \\
\hline 7. Actividades Sociais & $-.267^{* *}$ & $-.255^{* *}$ & $-.262^{* *}$ & $.563^{* *}$ & $.426^{* *}$ & $.329 * *$ & - \\
\hline$M$ & 8.304 & 8.984 & 8.660 & 21.586 & 16.345 & 12.693 & 10.288 \\
\hline$D P$ & 3.110 & 2.897 & 2.908 & 3.247 & 3.076 & 2.469 & 3.143 \\
\hline
\end{tabular}

Nota: **. A correlação é significativa no nível de $p \leq .01$ (2 extremidades). $M=$ Média; $D P=$ Desvio-padrão

Foram realizadas análises de variância multivariada (MANOVA) da satisfação com o suporte social e do funcionamento familiar, com o intuito de verificar as diferenças entre elas, em função da idade dos adolescentes (Quadro 3). Estes foram divididos em dois grupos de idade (dos 15 aos 16 anos e dos 17 aos 20 anos). Actualmente, o período de transição da infância para a idade adulta ocupa uma parte mais abrangente ao longo da vida. Em vez de 10 a 19 anos de idade, corresponde às idades de 10 a 24 anos (Sawyer et al., 2018). 
Assim, enquanto o primeiro grupo insere-se no primeiro ano de adaptação no ensino secundário, o segundo grupo insere-se numa fase mais próxima da idade adulta. De acordo com os resultados obtidos, verifica-se que não existem diferenças significativas na satisfação com o suporte social em função da idade dos adolescentes $\left[\mathrm{F}_{(4,360)}=2.147 ; p=.075\right]$.

No que diz respeito, ao funcionamento familiar em função da idade dos adolescentes, os resultados das indicam que não existem diferenças estatisticamente significativas $\left[\mathrm{F}_{(3,361)}=2.617, p=.051\right]$.

Quadro 3

Análise diferencial da satisfação com o suporte social e do funcionamento familiar em função da idade dos adolescentes.

\begin{tabular}{lccc}
\cline { 2 - 4 } & $\begin{array}{c}{[15-16 \text { anos }](1)} \\
\mathrm{M} \pm \mathrm{DP}\end{array}$ & $\begin{array}{c}{[17-20 \text { anos }](2)} \\
\mathrm{M} \pm \mathrm{DP}\end{array}$ & $p$ value \\
\hline ESSS & & & \\
Satisfação com os amigos & $21.907 \pm 2.930$ & $21.180 \pm 3.578$ & n.s. \\
Intimidade & $16.525 \pm 2.869$ & $16.118 \pm 3.315$ & n.s. \\
Satisfação com a família & $12.995 \pm 2.221$ & $12.311 \pm 2.712$ & n.s. \\
Atividades Sociais & $10.407 \pm 3.026$ & $10.137 \pm 3.287$ & n.s. \\
\hline SCORE-15 & & & \\
Recursos Familiares & $8.147 \pm 3.000$ & $8.503 \pm 3.243$ & n.s. \\
Comunicação na Família & $8.745 \pm 2.704$ & $9.286 \pm 3.105$ & n.s. \\
Dificuldades Familiares & $8.294 \pm 2.742$ & $9.124 \pm 3.051$ & n.s. \\
\hline
\end{tabular}

Nota: ESSS = Escala de Satisfação com o Suporte Social; SCORE-15 = Systemic Clinical Outcome and Routine Evaluation; $M=$ Média; $D P=$ Desvio-padrão; n.s. = não significativo

Foram realizadas análises de variância multivariada (MANOVA) da satisfação com o suporte social e do funcionamento familiar, em função da variável configuração familiar dos adolescentes (Quadro 4).

Relativamente à satisfação do suporte social em função da variável configuração familiar, verificou-se que existem diferenças significativas $\left[\mathrm{F}_{(4,360)}=4.264 ; p=.002 ; \eta^{2}=.045\right]$, nomeadamente, na dimensão satisfação com a família $\left[\mathrm{F}_{(1,363)}=15.402 ; p=<.001 ; \eta^{2}=\right.$ .041], sendo que adolescentes de famílias com pais casados ou em união de facto apresentam maiores níveis de satisfação com a família $(M=$ 12.868; $D P=2.330$ ) relativamente aos adolescentes com os pais divorciados $(M=11.275$; $D P=3.080$ ).

No que diz respeito ao funcionamento familiar em função da variável configuração familiar, verificou-se que existem diferenças significa- tivas $\left[\mathrm{F}_{(3,361)}=6.086 ; p=<.001 ; \eta^{2}=.048\right]$. Essas diferenças estão presentes em todas as dimensões, recursos familiares $[\mathrm{F}(1,363)$ $\left.=6.184 ; p=.013 ; \eta^{2}=.017\right]$ sendo que os adolescentes com pais divorciados $(M=9.450$; $D P=3.351)$ apresentam maiores dificuldades de recursos familiares quando comparados aos adolescentes cujos pais se encontram casados ou em união de facto $(M=8.163 ; D P=3.055)$. Assim como, a dimensão comunicação na família, $\left[\mathrm{F}_{(1,363)}=7.093 ; p=.008 ; \eta^{2}=.019\right]$, sendo que os adolescentes com pais divorciados $(M=10.125 ; D P=3.073)$ apresentam maiores dificuldades de comunicação na família quando comparados aos adolescentes cujos pais se encontram casados ou em união de facto $(M=$ 8.843; $D P=2.847)$. Por fim, na dimensão dificuldades familiares $\left[\mathrm{F}_{(1,363)}=18.329 ; p=.000\right.$; $\left.\eta^{2}=.048\right]$, os adolescentes com pais divorciados $(M=10.475 ; D P=2.909)$ apresentam 
maiores dificuldades familiares quando comparados aos adolescentes cujos pais se encontram casados ou em união de facto $(M=8.437 ; D P$ $=2.833$ ).

Quadro 4

Análise diferencial da satisfação com o suporte social e do funcionamento familiar em função da configuração familiar dos adolescentes.

\begin{tabular}{lccc}
\cline { 2 - 4 } & $\begin{array}{c}\text { Pais casados }(1) \\
\mathrm{M} \pm \mathrm{DP}\end{array}$ & $\begin{array}{c}\text { Pais Divorciados } \\
\mathrm{M} \pm \mathrm{DP}\end{array}$ & $p$ values \\
\hline ESSS & & & n.s. \\
Satisfação com os amigos & $21.646 \pm 3.171$ & $21.100 \pm 3.822$ & n.s. \\
Intimidade & $16.394 \pm 3.092$ & $15.950 \pm 2.952$ & $<.001 \mid 1>2$ \\
Satisfação com a família & $12.868 \pm 2.330$ & $11.275 \pm 3.080$ & n.s. \\
Actividades Sociais & $10.329 \pm 3.065$ & $9.950 \pm 3.741$ & p grupos \\
\hline SCORE-15 & & & $.013 \mid 1<2$ \\
Recursos Familiares & $8.163 \pm 3.055$ & $9.450 \pm 3.351$ & $.008 \mid 1<2$ \\
Comunicação na Família & $8.843 \pm 2.847$ & $10.125 \pm 3.073$ & $<.001 \mid 1<2$ \\
Dificuldades Familiares & $8.437 \pm 2.833$ & $10.475 \pm 2.909$ & \\
\hline
\end{tabular}

Nota: ESSS = Escala de Satisfação com o Suporte Social; SCORE-15 = Systemic Clinical Outcome and Routine Evaluation; $M=$ Média; $D P=$ Desvio-padrão; n.s. = não significativo

Foram realizadas análises de variância multivariada (MANOVA) da satisfação com o suporte social e do funcionamento familiar, com o intuito de verificar as diferenças entre elas, em função do sexo dos adolescentes (Quadro 5).

Não se verificaram diferenças significativas na satisfação com o suporte social em função da variável sexo $\left[\mathrm{F}_{(4,360)}=1.342 ; p=.254\right]$.

Relativamente ao funcionamento familiar, também não se verificou diferenças significativas $\left[\mathrm{F}_{(3,361)}=1.808 ; p=.145\right]$.

Quadro 5

Análise Diferencial da Satisfação com o Suporte Social e do Funcionamento Familiar em Função do Sexo dos Adolescentes.

\begin{tabular}{|c|c|c|c|}
\hline & \multirow{3}{*}{$\begin{array}{c}\text { Feminino (1) } \\
\mathrm{M} \pm \mathrm{DP}\end{array}$} & \multirow{3}{*}{$\begin{array}{c}\text { Masculino (2) } \\
\mathrm{M} \pm \mathrm{DP}\end{array}$} & \multirow{3}{*}{$p$ values } \\
\hline & & & \\
\hline & & & \\
\hline \multicolumn{4}{|l|}{ ESSS } \\
\hline Satisfação com os amigos & $21.512 \pm 3.384$ & $21.691 \pm 3.054$ & n.s. \\
\hline Intimidade & $16.493 \pm 2.945$ & $16.138 \pm 3.249$ & n.s. \\
\hline Satisfação com a família & $12.704 \pm 2.486$ & $12.678 \pm 2.454$ & n.s. \\
\hline Actividades Sociais & $10.150 \pm 3.133$ & $10.480 \pm 3.156$ & n.s. \\
\hline \multicolumn{4}{|l|}{ SCORE-15 } \\
\hline Recursos Familiares & $8.211 \pm 3.011$ & $8.434 \pm 3.250$ & n.s. \\
\hline Comunicação na Família & $8.704 \pm 2.685$ & $9.375 \pm 3.137$ & n.s. \\
\hline Dificuldades Familiares & $8.507 \pm 2.828$ & $8.875 \pm 3.013$ & n.s. \\
\hline
\end{tabular}

Nota: ESSS = Escala de Satisfação com o Suporte Social; SCORE-15 = Systemic Clinical Outcome and Routine Evaluation; $M=$ Média; $D P=$ Desvio-padrão; n.s. = não significativo 
Foram realizadas análises de variância multivariada (MANOVA) da satisfação com o suporte social e do funcionamento familiar, em função do tipo de fratria dos adolescentes (Quadro6).

Os resultados da análise multivariada (MANOVA) para a satisfação com o suporte social revelaram que não existem diferenças estatisticamente significativas consoante $\mathrm{o}$ número de irmãos que os adolescentes têm $\left[\mathrm{F}_{(8,718)}=1.628 ; p=.113\right]$.

No respeitante ao funcionamento familiar em função do tipo de fratria a que pertencem os adolescentes, os resultados também revelaram a não existência de diferenças significativas $\left[\mathrm{F}_{(6,720)}=.853, p=.530\right]$.

Quadro 6

Análise diferencial da Satisfação com o Suporte Social e do Funcionamento Familiar em Função do Tipo de Fratria dos Adolescentes.

\begin{tabular}{lcccc}
\cline { 2 - 5 } & $\begin{array}{c}\text { Filho único (1) } \\
\mathrm{M} \pm \mathrm{DP}\end{array}$ & $\begin{array}{c}\text { Com 1 irmão (2) } \\
\mathrm{M} \pm \mathrm{DP}\end{array}$ & $\begin{array}{c}\text { Com 2 ou mais irmãos (3) } \\
\mathrm{M} \pm \mathrm{DP}\end{array}$ & $p$ values \\
\hline ESSS & & & & \\
Satisfação com os amigos & $21.012 \pm 3.733$ & $21.757 \pm 3.069$ & $21.750 \pm 3.121$ & n.s. \\
Intimidade & $16.060 \pm 3.607$ & $16.481 \pm 2.857$ & $16.278 \pm 3.050$ & n.s. \\
Satisfação com a família & $12.241 \pm 2.827$ & $13.014 \pm 2.108$ & $12.278 \pm 2.869$ & n.s. \\
Actividades Sociais & $10.193 \pm 3.046$ & $10.262 \pm 3.166$ & $10.472 \pm 3.220$ & n.s. \\
\hline SCORE-15 & & & & \\
Recursos Familiares & $8.361 \pm 3.259$ & $8.095 \pm 2.972$ & $8.847 \pm 3.300$ & n.s. \\
Comunicação na Família & $9.072 \pm 2.849$ & $8.752 \pm 2.821$ & $9.556 \pm 3.117$ & n.s. \\
Dificuldades Familiares & $8.590 \pm 3.220$ & $8.538 \pm 2.824$ & $9.097 \pm 2.769$ & n.s. \\
\hline
\end{tabular}

Nota: ESSS = Escala de Satisfação com o Suporte Social; SCORE-15 = Systemic Clinical Outcome and Routine Evaluation; $M=$ Média; $D P=$ Desvio-padrão; n.s. = não significativo

\section{DISCUSSÃO}

PO presente estudo pretendeu averiguar a relação entre a satisfação com o suporte social e o funcionamento familiar numa amostra de adolescentes com e sem irmãos. Adicionalmente, de uma forma mais específica, pretendeu-se apurar as possíveis diferenças face ao suporte social e ao funcionamento familiar dos adolescentes tendo em conta a idade, o sexo, a configuração familiar e o tipo de fratria.

Os adolescentes inquiridos apresentam níveis de satisfação com o suporte social (com os amigos, família, intimidade e com as actividades sociais) que se associam de forma negativa com o funcionamento familiar, isto significa que à medida que aumenta a satisfação com o suporte social dos adolescentes tendem a diminuir as dificuldades do funcionamento familiar, ao nível dos recursos familiares e da comunicação da família, e vice-versa. Durante a adolescência, o papel da família torna-se fundamental devido às mudanças que ocorrem nesta fase $\mathrm{e}$ as relações familiares podem ser consideradas como factores protectores para os membros da família (Seibel et al., 2017). No entanto, durante esta fase de mudança, os relacionamentos com os amigos/parceiros expandem-se e ajudam os adolescentes a desenvolverem a sua autonomia (Wrzus et al., 2013). Uma vez mais, sendo que a comunicação familiar e as dificuldades familiares são dois aspetos fundamentais do funcionamento familiar, é natural que quanto maior sejam as dificuldades, a comunicação piore (Wang \& Zhao, 2013). Deste modo, verificou-se através de um estudo que o funcionamento familiar apresentou uma associação negativa com o suporte social (Seibel et al., 2017). 
Relativamente à satisfação com o suporte social, não se observaram diferenças significativas em função da idade dos adolescentes. Não corroborando os nossos resultados, num estudo de Antunes e Fontaine (2005), com adolescentes do ensino secundário apurou-se que adolescentes mais novos tinham uma maior percepção do suporte social em geral, comparativamente com os adolescentes mais velhos. De facto, e para Sarriera et al. (2007) de acordo com o aumento da faixa etária, a possibilidade de autonomia torna-se maior. No entanto, o fator condicionante para os adolescentes mais velhos diz respeito à falta de tempo, uma vez que estando mais próximos da vida adulta, com os estudos ou com o emprego o tempo torna-se escasso. Assim, os adolescentes mais novos dispõem de mais tempo e estão mais condicionados ao controlo da família em relação aos mais velhos.

No que diz respeito, ao funcionamento familiar dos adolescentes, não existem diferenças estatisticamente significativas entre os grupos de adolescentes em função da sua idade. No entanto, a literatura indica que na adolescência existe uma maior prevalência de famílias com dificuldades no funcionamento familiar, em adolescentes com idades mais próximas da idade adulta, em relação a famílias com adolescentes mais novos (Prioste, Tavares, \& Magalhães, 2019).

Relativamente à satisfação com o suporte social em função do sexo dos adolescentes, não existem diferenças significativas. Segundo Rabaglietti e Ciairano (2008), a percepção do suporte social dos amigos não é linear ao longo do tempo. No início da adolescência, ocorrem mais conflitos entre os jovens, no entanto, estes sentem-se mais apoiados por parte dos seus amigos, em comparação com os jovens que se encontram no final da adolescência. É notória uma diminuição da percepção do suporte social da família e dos amigos ao longo do tempo, porém verifica-se que os adolescentes do sexo feminino têm uma maior percepção de suporte social proveniente de amigos e colegas de turma, em relação aos do sexo masculino (Rabaglietti \& Ciairano, 2008). Segundo Alves e Dell'Aglio (2015), existem diferenças por sexo na percepção do suporte social, sendo que os indivíduos do sexo feminino perceberam maior suporte social do que os do sexo masculino (Alves \& Dell'Aglio, 2015). Também, um estudo de Ikiz e Cakar (2010) afirma que são os indivíduos do sexo feminino que apresentam maior percepção do suporte social dos amigos comparativamente com os do sexo masculino.

No que diz respeito ao funcionamento familiar, os resultados sugerem não haver diferenças de sexo quanto à forma como os adolescentes o percepcionam, indo ao encontro da literatura existente. De facto, o estudo de Coppari, Eguiarte, Zavala e Bagnoli (2017) procurou explorar diferenças em função do sexo dos adolescentes quanto ao funcionamento familiar, e verificou-se que os adolescentes de ambos os sexos percebiam o funcionamento familiar de forma similar.

No que respeita aos resultados averiguados ao nível das diferenças em função da configuração familiar a que pertencem os adolescentes, foi possível verificar que adolescentes de famílias intactas (pais casados ou em união de facto) apresentam níveis superiores de satisfação com a família relativamente aos adolescentes provenientes de famílias com pais divorciados. Segundo Silva, Melo e Mota (2016) os jovens de famílias intactas possuem uma melhor percepção do suporte da família, comparando com os jovens de famílias que passaram por separação ou divórcio. O processo de divórcio e separação pode estar associado a conflitos, o que virá a interferir na estabilidade relacional familiar, o que pode diminuir as trocas afectivas e a consequente percepção das mesmas (Hack \& Ramires, 2010). Os adolescentes de pais divorciados tendem a apresentar mais dificuldades no campo emocional, comportamental e social (Amato \& Anthony, 2014). Verificou-se ainda que existem diferenças significativas em 
todas as dimensões do funcionamento familiar, isto é, recursos familiares, comunicação na família e dificuldades familiares, sendo que os adolescentes provenientes de famílias com pais divorciados apresentam níveis superiores de dificuldades familiares, quando comparados aos adolescentes provenientes de famílias onde os pais estão casados ou em união de facto. Este resultado corrobora um estudo realizado, em que o objectivo era investigar as diferenças existentes no funcionamento familiar, entre as tipologias de configuração familiar, e concluíram que o funcionamento familiar era melhor nas famílias nucleares intactas do que nas outras configurações familiares (Shek, Xie, $\&$ Lin, 2015).

Relativamente à satisfação com o suporte social em função do tipo de fratria a que os adolescentes pertencem verificou-se a inexistência de diferenças significativas consoante o número de irmãos que os adolescentes têm. Estes dados não corroboram estudos anteriores, num estudo de Cruz, Nelas, Chaves, Almeida e Costa (2016), em que se apurou que a variável número de irmãos interfere estatisticamente com o suporte social. Num outro estudo, sobre o suporte social verificou-se que os irmãos desempenham um papel importante, sobretudo com irmãos da mesma idade (Wal cka-Matyja, 2015). É importante realçarmos que, a fonte de suporte social mais importante para o desenvolvimento do adolescente é o meio familiar (Kong \& You, 2013). A literatura citada por Fernandes (2005) refere que os filhos únicos têm mais dificuldades de se relacionarem com os outros, comparativamente com os indivíduos que têm irmãos.

Relativamente ao funcionamento familiar em função do tipo de fratria a que pertencem os adolescentes, os nossos resultados também revelaram a inexistência de diferenças significativas. A literatura sugere que com a chegada de um filho, ocorrem implicações para todos os membros da família, incluindo os irmãos, uma vez que se dá em diferentes momentos da vida familiar (Velásquez, 1997). No entanto, as diferentes experiências na fratria não são apenas em função da diferença de ordem de nascimento, mas também do sexo, do número de irmãos e da idade entre eles (Fernandes, 2002). É na relação com a família, em particular com os irmãos, que começa o desenvolvimento social do indivíduo. A família tem um impacto significativo e uma forte influência no comportamento dos indivíduos (Dessen \& Polonia, 2007). Assim, um vínculo seguro com as figuras parentais concede aos filhos, a segurança e confiança suficiente para a construção de relações fraternas e o desenvolvimento de relacionamentos significativos (Fernandes, 2002).

\section{CONCLUSÃO}

O presente estudo procurou analisar a relação existente entre a satisfação com o suporte social e o funcionamento familiar em adolescentes, com e sem irmãos. Concluiu-se que a satisfação do suporte social se associou de forma negativa com o funcionamento familiar, isto significa que à medida que aumenta a satisfação com o suporte social dos adolescentes tendem a diminuir as dificuldades do funcionamento familiar, e vice-versa. Relativamente à satisfação com o suporte social, não se observaram diferenças significativas em função da idade dos adolescentes, consoante o número de irmãos que os adolescentes têm e em função do sexo dos adolescentes. No que diz respeito ao funcionamento familiar, verificou-se que não existem diferenças significativas entre os grupos de adolescentes em função da sua idade, sexo, assim como em função do tipo de fratria a que pertencem os adolescentes. Por outro lado, ao nível das diferenças em função da configuração familiar a que pertencem os adolescentes, foi possível verificar que adolescentes de famílias intactas (pais casados ou em união de facto) apresentam níveis superiores de satisfação com a família relativamente aos adolescentes provenientes de famílias com pais divorciados. 
Importa referir que não foi encontrado qualquer estudo, no contexto português, que analisasse a forma como a satisfação com o suporte social é influenciada pelo funcionamento familiar em adolescentes, com e sem irmãos. Assim sendo, o estudo foi pertinente, uma vez que se objectivou fornecer mais um contributo positivo à compreensão da associação que existe entre a satisfação com o suporte social e o funcionamento familiar, pelos adolescentes.

Deste modo, e tendo em conta os resultados obtidos, torna-se importante que mais investigações sejam desenvolvidas no sentido de contribuir para a qualidade das relações sociais e familiares, nesta fase do ciclo vital. Seria importante a criação de programas de intervenção em contexto escolar, visando alertar e sensibilizar os adolescentes, assim como respectivas famílias a melhorar o funcionamento familiar, assim como as relações entre os pares para que se tornem relações saudáveis, tendo como objectivo proporcionar aos adolescentes, um desenvolvimento saudável, bem como o sucesso escolar.

Após a conclusão do presente estudo importa referir algumas limitações, a principal é a falta de investigação prévia relativamente à satisfação com o suporte social e com o funcionamento familiar em função do número de irmãos, dos adolescentes. Esta limitação levanta a necessidade e a sugestão de serem desenvolvidos outros estudos sobre o tema, principalmente em Portugal. Acrescenta-se, ainda, o facto do tamanho da amostra, que não é representativa da população portuguesa, uma vez que a informação foi recolhida apenas em duas escolas da região norte do país. Assim, esta amostra pode ter características sociais e culturais específicas ao meio em que se encontra inserida. Além disso, por se tratar de um questionário de auto-relato extenso poderá levar à fadiga dos adolescentes, comprometendo o preenchimento do mesmo.

Em suma, sugere-se a realização de novos estudos que consigam colmatar aspectos menos positivos desta investigação, ou seja, em investigações futuras seria importante aumentar o tamanho da amostra, e estender este mesmo estudo em diferentes meios. Seria ainda pertinente desenvolverem-se estudos mais aprofundados que conciliem metodologias quantitativas e qualitativas.

Por último, a presente investigação procurou dar um contributo para se compreender melhor como o funcionamento familiar favorece a construção de uma rede de relações e de experiências ao longo do ciclo de vida, e especificamente nos adolescentes, contribuindo para o seu bem-estar.

Agradecimentos:

Nada declarado.

\section{Conflito de Interesses:}

Nada declarado.

Financiamento:

Nada declarado.

\section{REFERÊNCIAS}

Adler-Constantinescu, C., Besu, E. C., \& Negovan, V. (2013). Perceived social support and perceived self-efficacy during adolescence. Procedia - Social and Behavioral Sciences, 78, 275-279. doi: 10.1016/j. sbspro.2013.04.294

Alves, C. F., \& Dell'Aglio, D. D. (2015). Percepção de apoio social de adolescentes de escolas públicas. Revista de Psicologia da IMED, 7(2), 89-98. doi: 10.18256/21755027/psico-imed.v7n2p89-98

Almeida, L. S., \& Freire, T. (2017). Metodologia da investigação em psicologia e educação ( $5^{\mathrm{a}} \mathrm{Ed}$. Revista). Braga: Psiquilíbrios Edições.

Amato, P. R., \& Anthony, C. J. (2014). Estimating the effects of parental divorce and death with fixed effects models. Journal of Marriage and Family, 76(2), 370-386. doi:10.1111/ jomf. 12100 
Antunes, A., \& Fontaine, A. M. (2005). Percepção de apoio social na adolescência: Análise fatorial confirmatória da Escala Social Support Appraisals. Paidéia, 15(32), 355-366. doi: 10.1590/S0103-863X2005000300005

Belanger, N. M. S., \& Patrick, J. H. (2018). The influence of source and type of support on college students' physical activity behavior. Journal of Physical Activity and Health, 15(3), 183-190. doi: 10.1123/jpah.2017-0069

Berge, J. M., Wall, M., Larson, N., Loth, K. A., \& Neumark-Sztainer, D. (2013). Family functioning: Associations with weight status, eating behaviors, and physical activity in adolescents. Journal Adolescent Health, 52(3), 351-357. doi: 10.1016/j. jadohealth.2012.07.006

Chen, W., Zhang, D., Pan, Y., Hu, T., Liu, G., \& Luo, S. (2017). Perceived social support and self-esteem as mediators of the relationship between parental attachment and life satisfaction among Chinese adolescents. Personality and Individual Differences, 108, 98-102. doi: 10.1016/j.paid.2016.12.009

Cohen, J. W. (1988). Statistical power analysis for the behavioral sciences (2nd ed.). Hillsdale, $\mathrm{NJ}$ : Lawrence Erlbaum Associates.

Coob, S. (1976). Social support as a moderator of life stress. Psychosomatic Medicine, 38(5), 300-314. doi: 10.1097/00006842197609000-00003

Coparri, N., Eguiarte, B. E. B., Zavala, G. C., \& Bagnoli, L. (2017). Differences on the perception of family functioning in adolescents from Paraguay and Mexico. Salud \& Sociedad, 8(2), 126-135. doi: 10.22199/ S07187475.2017.0002.00002

Cox, J. M. (2010). Family systems and sibling relationships. Child Development Perspectives, 4(2), 95-96. doi:10.1111/j.1750-8606.2010. 00124.x

Cruz, C., Nelas, T., Chaves, C., Almeida, M., \& Costa, S. (2016). O suporte social dos estudantes do ensino superior. INFAD - Revista de Psicologia, 2(1), 81-88. doi: 10.17060/ijodaep.2016

Cutrona, C. E., \& Russell, D. W. (1990). Type of social support and specific stress: Toward a theory of optimal matching. In B. R. Sarason, I. G. Sarason, \& G. G. Pierce (Ed.), Social support: An interactional view (pp. 454-481). New York: John Wiley \& Sons.

Dessen, M. A., \& Polonia, A. C. (2007). A família e a escola como contextos de desenvolvimento humano. Paidéia, 17(36), 21-32. doi: 10.1590/S0103-863X2007000100003

Emam, M. M., \& Abu-Serei, U. S. (2014). Family functioning predictors of self-concept and self-esteem in children at risk for learning disabilities in Oman: Exclusion of parent and gender contribution. International Education Studies,7(10), 89-99. doi: 10.5539/ ies.v7n10p89

Fernandes, O. M. (2002). Semelhanças e diferenças entre irmãos. Lisboa: Climepsi.

Fernandes, O. M. (2005). Ser único ou ser irmão. Cruz Quebrada: Oficina do Livro.

Guerreiro, D. F., \& Sampaio, D. (2013). Comportamentos autolesivos em adolescentes: Uma revisão. Revista Portuguesa de Saúde Pública, 31(2), 213-222. doi: 10.1016/j.rpsp.2013.05.001

Hack, S., \& Ramires, V. (2010). Adolescência e divórcio parental: Continuidades e rupturas dos relacionamentos. Psicologia Clínica, 22(1), 85- 97. doi:10.1590/S010356652010000100006

Ikiz, F., \& Cakar, F. (2010). Perceived social support and self-esteem in adolescence. Procedia Social and Behavioral Sciences, 5, 23382342. doi:10.1016/j.sbspro.2010.07.460

Kong, F., \& You, X. (2013). Loneliness and selfesteem as mediators between social support and life satisfaction in late adolescence. Social Indicators Research, 110(1), 271-279. doi: 10.1007/s11205-011-9930-6

Lamb, A. E., Biesecker, B. B., Umstead, K. L., Muratori, M., Biesecker, L. G., \& Erby, L. H. (2016). Family functioning mediates adaptation in caregivers of individuals 
with Rett syndrome. Patient Education and Counseling, 99(11), 1873-1879. doi: 10.1016/j.pec.2016.06.018

Matejevic, M., Jovanovic, D., \& Lazarevic, V. (2014). Functionality of family relationships and parenting style in families of adolescents with substance abuse problems. Procedia Social and Behavioral Sciences, 128, 281-287. doi: 10.1016/j.sbspro.2014.03.157

Marôco, J. (2014). Análise de equações estruturais. Fundamentos teóricos, software e aplicações. Lisboa: ReportNumber.

Marôco, J. P., Campos, J. A. D. B., Vinagre, M.G., \& Pais-Ribeiro, J.L. (2014). Adaptação transcultural Brasil-Portugal da Escala de Satisfação com o Suporte Social para estudantes do ensino superior. Psicologia: Reflexão e Crítica, 27(2), 247-256. doi: 10.1590/1678-7153.201427205

Matsumoto, S., Yamaoka, K., Takahashi, K., Tanuma, J., Mizushima, D., Do, C. D., Nguyen, D. T., Nguyen, H. D. T., Van Nguyen, K., \& Oka, S. (2017). Social support as a key protective factor against depression in HIV-Infected patients: Report from large HIV clinics in Hanoi, Vietnam. Scientific Reports, 7(1), 1-12. doi:10.1038/s41598017-15768-w

Mattson, M., \& Hall, J. G. (2011). Linking health communication with social support. In $\mathrm{M}$. Mattson \& J. G. Hall (1st Ed.), Health as communication nexus: $A$ service-learning approach (pp. 181-218). Dubuque, IA: Kendall Hunt Publishing Company.

Mühlen, M. C. V., Stechman, M. F., Aerts, D. G., Palazzo, L., Alves, G. G., \& Câmara, S. G. (2013). Estratégias de enfrentamento de situações estressantes e satisfação com a vida entre adolescentes escolares no sul do brasil. Revista de Iniciação Científica, 11, 77-85.

Pais-Ribeiro, J.L. (1999). Escala da Satisfação com o Suporte Social (ESSS). Análise Psicológica, 3(17), 547-558.

Pallant, J. F. (2005). SPSS survival manual: A step by step guide to data analysis using SPSS for Windows (Version 12). Sydney: Allen \& Unwin.

Paulsen, V., \& Berg, B. (2016). Social support and interdependency in transition to adulthood from child welfare services. Children and Youth Services Review, 68, 125-131. doi:10.1016/j. childyouth.2016.07.006

Portugal, A., Sotero, L., Cunha, D., Vilaça, M., \& Relvas, A. P. (2010, Outubro). SCORE15: Exploratory study of preliminary data in a sample of Portuguese families. Comunicação apresentada no $7^{\circ}$ European Family Therapy Association Congress, Paris, France.

Prioste, A., Tavares, P., \& Magalhães, E. (2019). Tipologias de funcionamento familiar: Do desenvolvimento identitário à perturbação emocional na adolescência e adultez emergente. Análise Psicológica, 2(37), 173-192. doi: 10.14417/ap.1534

Rabaglietti, E., \& Ciairano, S. (2008). Quality of friendship relationships and developmental tasks in adolescence. Cognition, Brain, Behavior, 12(2), 183-203.

Sangalang, C.C., \& Gee, G. C. (2012). Depression and anxiety among asian americans: The effects of social support and strain. National Association of Social Workers, 57(1), 49-60. doi:10.1093/sw/swr005

Sarriera, J. C., Paradiso, A. C., Mousquer, P. N., Marques, L. F., Hermel, J. S., \& Coelho, R P. S. (2007). Significado do tempo livre para adolescentes de classe popular. Psicologia: Ciência e Profissão, 27(4), 718-729. doi:10.1590/S1414-98932007000400012

Sawyer, S. M., Azzopardi, P. S., Wickremarathne, D., \& Patton, G. C. (2018). The age of adolescence. Lancet Child Adolescent Health, 2(3), 1-6. doi: 10.1016/S23524642(18)30022-1

Schulte, M. T., Armistead, L., Marelich, W. D., Payne, D. L., Goodrum, N. M., \& Murphy, D. A. (2017). Maternal parenting stress and child perception of family functioning among families affected by HIV. Journal of the Association of Nurses in AIDS Care, 28(5), 
784-794. doi:10.1016/j.jana.2017.05.004

Seibel, B. L., Falceto, O. G., Hollist, C. S., Springer, P., Fernandes, C. L. C., \& Koller, S. H. (2017). Rede de apoio social e funcionamento familiar: Estudo longitudinal sobre famílias em vulnerabilidade social. Pensando Famílias, 21 (1), 120-136.

Shek, D., Xie, Q., \& Lin, L. (2015). The impact of family intactness on family functioning, parental control, and parent-child relational qualities in a Chinese context. Frontiers in Pediatrics, 2(149), 1-7. doi: 10.3389/ fped.2014.00149

Silva, A. R., Melo, O., Mota, A.C. (2016). Suporte social e individuação em jovens de diferentes configurações familiares. Temas em Psicologia, 24(4), 1311-1327. doi: 10.9788/ TP2016.4-07

Stratton, P., Bland, J., Janes, E., \& Lask, J. (2010). Developing an indicator of family function and a practicable outcome measure for systemic family and couple therapy: the SCORE. Journal of Family Therapy, 32(3), 232-258. doi: 10.1111/j.1467 6427.2010.00507.x.

Studzinski, N. G. (2016). Fatores de vulnerabilidade que influenciam a conduta infracional na adolescência: Uma revisão. Revista Adolescência e Conflitualidade, 15, 62-69.

Thompson, R. A. (2015). Social support and child protection: Lessons learned and learning. Child Abuse \& Neglect, 41, 19-29. doi: 10.1016/j.chiabu.2014.06.011

Tolou-Shams, M., Brogan, L., EspositoSmythers, C., Healy, M. G., Lowery, A., Craker, L., \& Brown, L. K. (2018). The role of family functioning in parenting practices of court involved youth. Journal of Adolescence, 63, 165-174. doi:10.1016/j. adolescence.2017.12.016
Velásquez, A. M. Q. (1997). Cambios en la dinámica familiar durante la gestación y el posparto. Investigación y Educación en Enfermaría, 15(1), 109-119.

Vilaça, M., Silva, J. T., \& Relvas, A. P. (2014). Systemic clinical outcome routine evaluation (SCORE-15). In A. P. Relvas \& S. Major (coord.), Instrumentos de avaliação familiar - Funcionamento e intervenção (pp. 23-41). Coimbra: Imprensa da Universidade de Coimbra.

Walęcka-Matyja, K. (2015). Relationships with siblings as a way of coping with stress in the early adulthood and the level of self-esteem. Polskie Forum Psychologiczne, 20(3), 285-304. doi: 10.14656/PFP20150301

Wang, J., \& Zhao, X. (2013). Perceived family functioning in depressed Chinese couples: A cross-sectional study. Nursing \& Health Sciences, 15(1), 9-14. doi: 10.1111/j.14422018.2012.00707.x

Wong, T. M. L., Branje, S. J.T., VanderValk, I. E., Hawk, S. T., \& Meeus, W. H. J. (2010). The role of siblings in identity development in adolescence and emerging adulthood. Journal of Adolescence, 33(5), 673-682. doi:10.1016/j. adolescence.2009.11.003

Wrzus, C., Hanel, M., Wagner, J., \& Neyer, F. J. (2013). Social network changes and life events across the life span: A meta-analysis. Psychological Bulletin, 139(1), 53-80. doi: 10.1037/a0028601 